\title{
Efficacy of different vinegars as antifungal agents against Cryptococuss neoformans and Sarocladium kiliense
}

\author{
Humaira Niamat*, Asmara Imtiaz, Muhammad Amjad Khan and \\ Muneeba \\ Department of Biology, Lahore Garrison University, Lahore, Pakistan \\ *Corresponding author's email: humairaniamat@lgu.edu.pk \\ Citation \\ Humaira Niamat, Asmara Imtiaz, Muhammad Amjad Khan and Muneeba. Efficacy of different vinegars as \\ antifungal agents against Cryptococuss neoformans and Sarocladium kiliense. Pure and Applied Biology. Vol. 9, \\ Issue 2, pp1646-1653. http://dx.doi.org/10.19045/bspab.2020.90173
}

Received: $17 / 12 / 2019$

Revised: 26/02/2020

Accepted: 10/03/2020

Online First: 24/03/2020

\section{Abstract}

Natural sources have caught the scientist's eye for the development of new active substances because of side effects of chemically synthetic preservatives and their ultimate hazardous effects on human health. In the present study, three types of vinegars; white distilled vinegar, grape vinegar and apple cider have been used to test the antifungal activity against Cryptococcus neoformans and Sarocladium kiliense. Rotten vegetables; cucumber and tomato were used to isolate these two fungal isolates. These isolates were identified using microscopic technique. Anti-fungal activity was observed followed by application of vinegars through agar well diffusion assay technique. All vinegars showed significant size of zone of inhibition against fungal specie. Distilled white vinegar showed the best effect against $C$. neoformans and S. kiliense forming zone of inhibition of $0.58 \pm 0.05 \mathrm{~mm}$ and $0.6 \pm 0.05 \mathrm{~mm}$. Grape vinegar forms a zone of inhibition of $0.52 \pm 0.05 \mathrm{~mm}$ and $0.48 \pm 0.05$, while $0.4 \pm 0.05 \mathrm{~mm}$ was observed against both fungal specie, as a result of application of apple cider. The results showed that vinegars can be used as an alternative to synthetic antifungal agents in order to overcome the drawbacks because they have active components i.e. phenolics and flavonoids etc. which have antifungal and anti-oxidant properties.

Keywords: Agar well diffusion; Anti-fungal agents; Apple cider; Cryptococcus neoformans; Distilled white vinegar; Grape Vinegar; Sarocladium kiliense

\section{Introduction}

Different antimicrobial agents have been in use for long time and these agents do not have any adverse effects. Many of the organic compounds are the antimicrobial metabolites of bacteria that are used to produce fermented foods. In fermentation process, raw materials are converted to products by the action of microorganisms and these products have acceptable qualities of food. Fermentation of food has a great economic value and these products have been contributed in improving the human health [1].
Scientific studies categorized the food borne pathogen behavior into two divisions, i.e., food science and medical field. Two different approaches are used to determine the behavior of food borne pathogens in food; commonly refers to as in vitro and in situ. In vitro studies aim at stimulating the behavior of food borne pathogen in laboratory giving a specific media for their growth, while in situ studies describe the microbial expression in real food under the conditions of storage and consumption of food [2]. 
C. neoformans contains two varieties namely C. neoformans var. grubii and C. neoformans var. neoformans. Colonies of $C$. neoformans are cream-colored and smooth, mucoid and have yeast like appearance of colonies. $C$. neoformans var. grubii is cosmopolitan in its distribution and this one cause $95 \%$ of all the C. neoformans infections. C. neoformans has been isolated from the menure of caged birds including canaries and parrots etc. $C$. neoformans var. grubii has also been isolated from the other environmental isolations including rotten or decomposed vegetables, fruits and fruit juices, wood dairy product and soil. The infections of $C$. neoformans var. neoformans are more prevalent in Europe including France, Italy and Denmark. Moreover, the infections are more related with older patients and may cause the skin infections [3]. The most infections associated to Cryptococcus are caused by the $C$. neoformans var. neoformans in humans [4]. C. neoformans is accountable for serious infections in patients with cellular immune defects, particularly patients with AIDS [5].

Sarocladium was formerly called Acremonium, but after study the taxonomy of Acremonium, some medically important species have been transferred to Sarocladium; i.e. S. kiliense (formerly A. kiliense) and $S$. strictum (formerly A. strictum). Morphologically these genera are similar, but they are phylogenetically distant. Sarocladium can be morphologically differentiated from Acremonium by its elongated phialides rising solitary on vegetative hyphae or on conidiophores that are sparsely or repeatedly branched, the production of abundant adelophialides and elongated conidia [6].

Different natural antimicrobials from the plant origin contain secondary metabolites having ability to inhibit or reduce the growth of bacteria, yeast and molds [7]. Plant essential oils have also been used as a flavoring agent in addition to its antimicrobial activity [8]. A large number of antimicrobial agents originated from animal origin and involved in host defense mechanisms [9]. Among the different natural products, vinegars are also contributing in its antimicrobial properties [10]. Vinegars i.e. Apple cider has an important element; maleic acid with bactericidal and fungicidal activity. Maleic acid has been reported for the treatment of various infections [11].

\section{Material and methods}

\section{Isolation and culturing of fungus}

The microorganisms to be isolated; were grown on the Potato dextrose agar media (containing 3.9g PDA in $100 \mathrm{ml}$ water). Rotten vegetables; cucumber and tomato were used to isolate the fungal specie. Small pieces of these were inoculated in the center of two different PDA plates. Then the plates were incubated at $37^{\circ} \mathrm{C}$ for 72 hours.

\section{Identification of fungal isolates}

After incubation period, two different fungal isolates were obtained. They were identified by microscopic technique. Fungal smear of each species was made and then stained with crystal violet stain. The slides were then observed under microscope.

\section{Agar-well diffusion method}

Principle: The antimicrobials present in the natural antifungal (vinegars) were allowed to diffuse out into the medium and interact in a plate freshly seeded with the test organisms. The resulting zones of inhibition were mostly circular in shape as there was a confluent lawn of growth. The diameter of zone of inhibition was measured in millimeters.

\section{Method to apply vinegars}

PDA pla4tes were prepared under aseptic conditions. Then, by using the borer, wells were made in the center of each plate. Three of the plates were swabbed with each of the two fungal isolates, using sterile cotton swabs. Then, $100 \mu$ of each vinegar i.e. apple cider, distilled white vinegar and grape vinegar were added in the wells. The plates were then incubated for 72 hours at $37^{\circ} \mathrm{C}$. The 
vinegars were allowed to diffuse out into the medium and interact in a plate freshly seeded with the test organisms. After incubation period, zones of inhibition were observed in each plate. The diameter of zone of inhibition was measured in millimeters with the help of Vernier caliper and diameter of each zone of inhibition was measured from three different directions. The mean values of zone of inhibition are mentioned in results.

\section{Results}

In present study, two different fungal species were isolated and identified on the basis of their colony morphological characteristics, color charts and microscopic examination. The isolated fungal species were $C$. neoformans and $S$. kiliense.

Morphological examination of fungal isolates (Fig. 1).
Microscopic examination of fungal isolates (Fig. 2).

Effect of distilled white vinegar on $C$. neoformans (Fig. 3).

Effect of grape vinegar on $C$. neoformans (Fig. 4).

Effect of apple cider on C. neoformans (Fig. $5)$.

Effect of distilled white vinegar on S. kiliense (Fig. 6).

Effect of grape vinegar on S. kiliense (Fig. 7). Effect of apple cider on S. kiliense (Fig. 8).

The best effect of distilled white vinegar against $C$. neoformans with mean value of $0.58 \pm 0.05 \mathrm{~mm}$ (Fig. 9).

The zones of inhibition of vinegars against $S$. kiliense (Fig. 10).

The varying zone of inhibition against fungal species (Table 1).

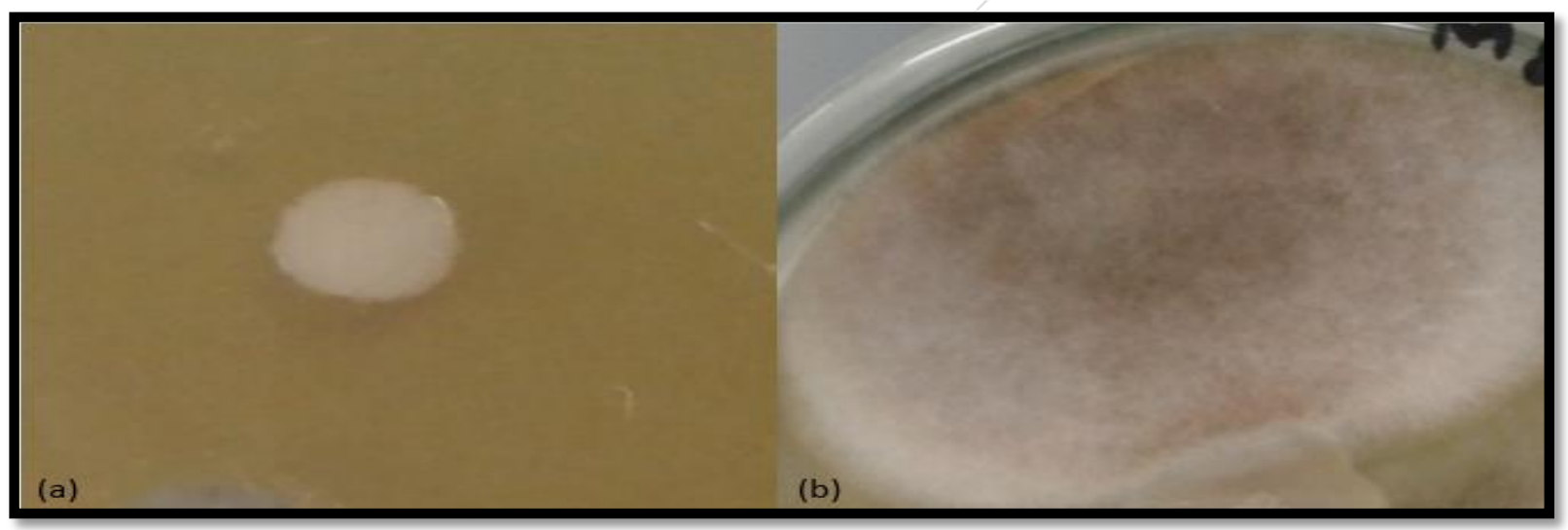

Figure 1. (a) Colony of C. neoformans (b) Colony of S. kiliense

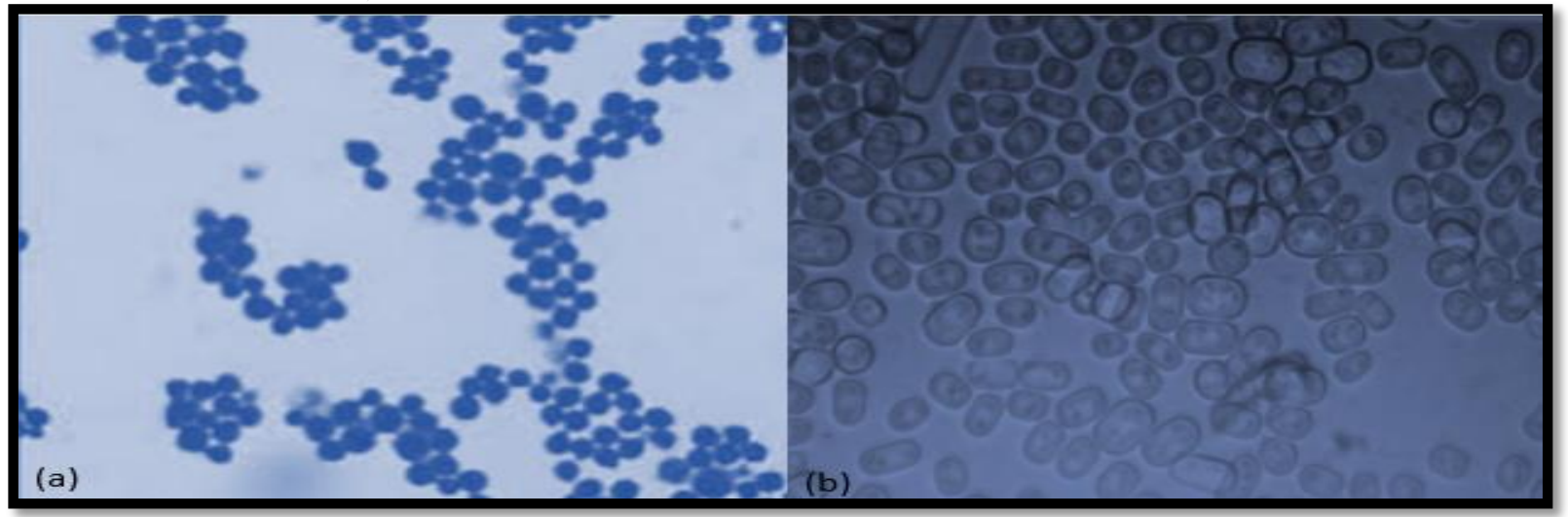

Figure 2 (a) Microscopic examination of $C$. neoformans (b) Microscopic examination of $S$. kiliense 


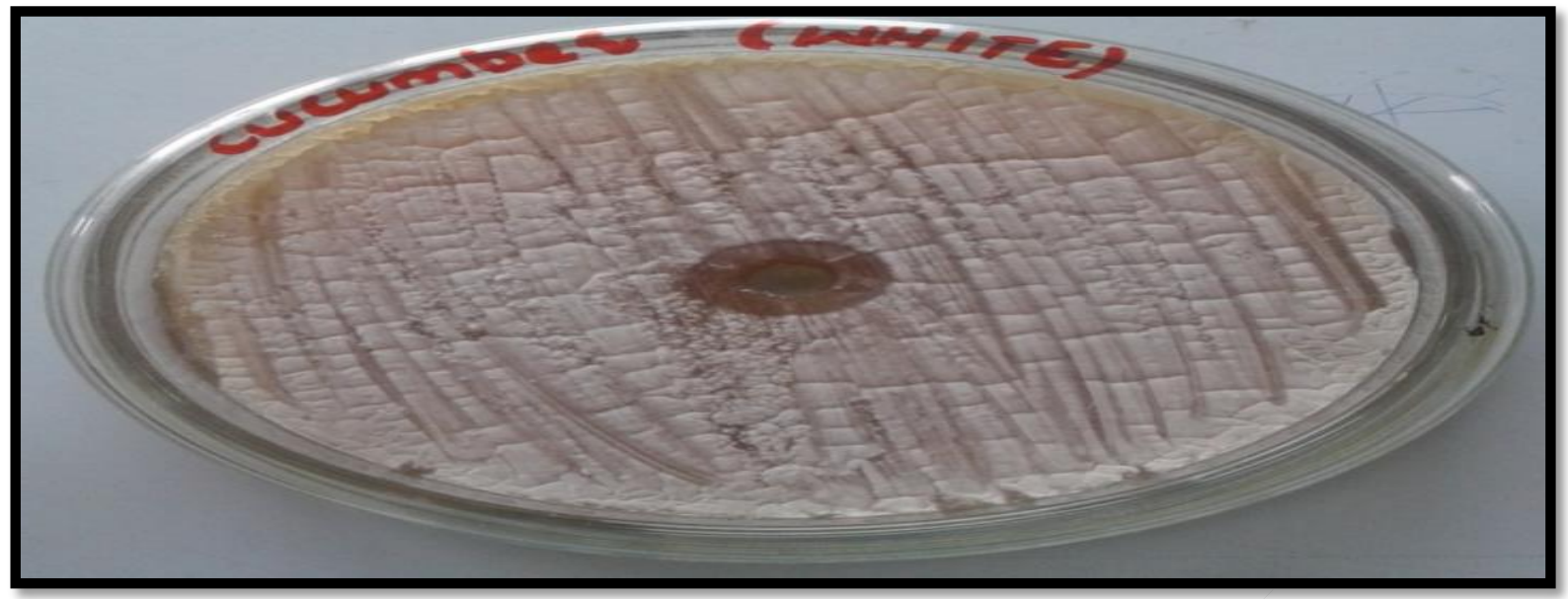

Figure 3. Effect of white vinegar on $C$. neoformans after 72 hours. Distilled white showed the activity against $C$. neoformans by forming zone of inhibition of $0.58 \pm 0.05 \mathrm{~mm}$ after 72 hours

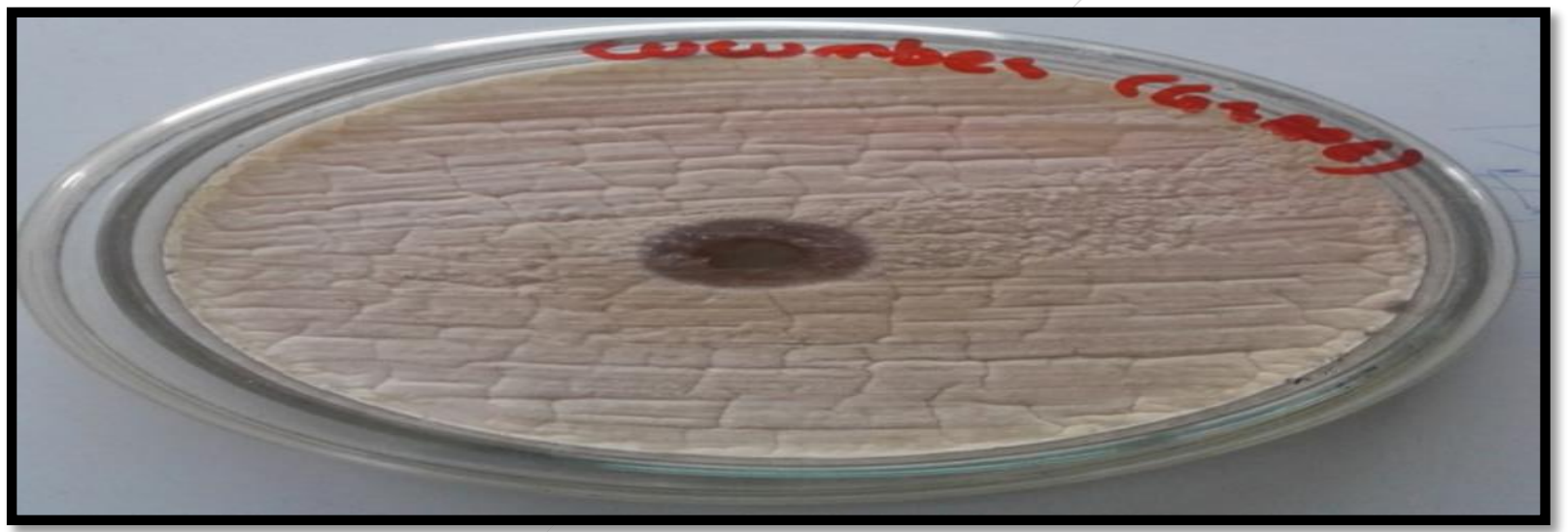

Figure 4. Effect of grape vinegar on $C$. neoformans after 72 hours. Grape vinegar showed the activity against $C$. neoformans by forming zone of inhibition of $0.52 \pm 0.05 \mathrm{~mm}$ after 72 hours

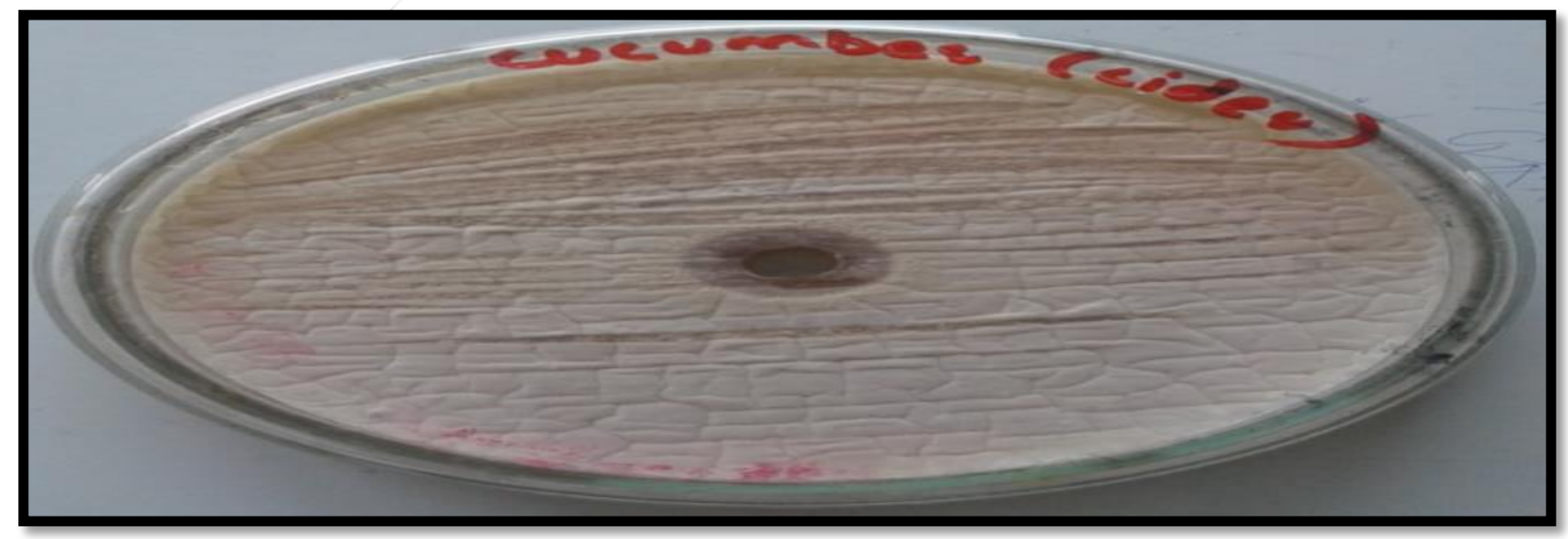

Figure 5. Effect of apple cider on $C$. neoformans after 72 hours. Apple cider vinegar shows activity against $C$. neoformans by forming zone of inhibition of $0.4 \pm 0.05 \mathrm{~mm}$ after 72 hours 


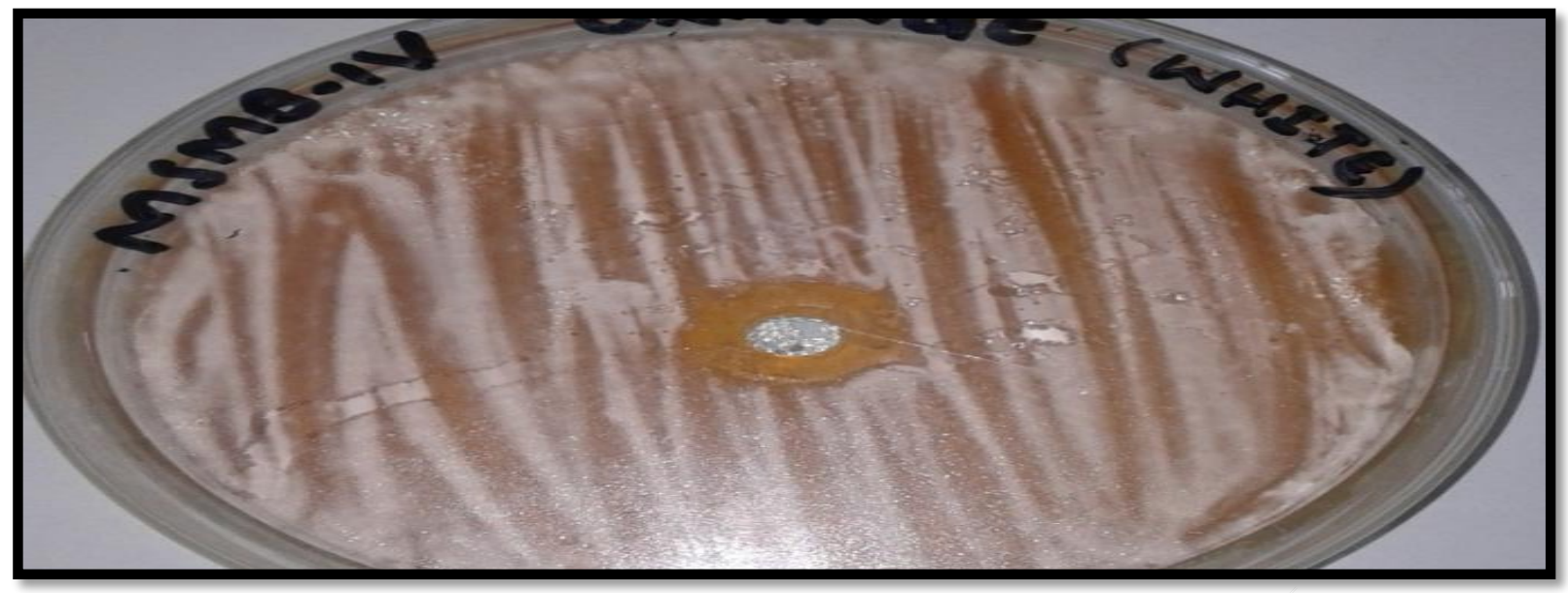

Figure 6. White vinegar showed the activity against $S$. kilense by forming zone of inhibition of $0.6 \pm 0.05 \mathrm{~mm}$ after 72 hours

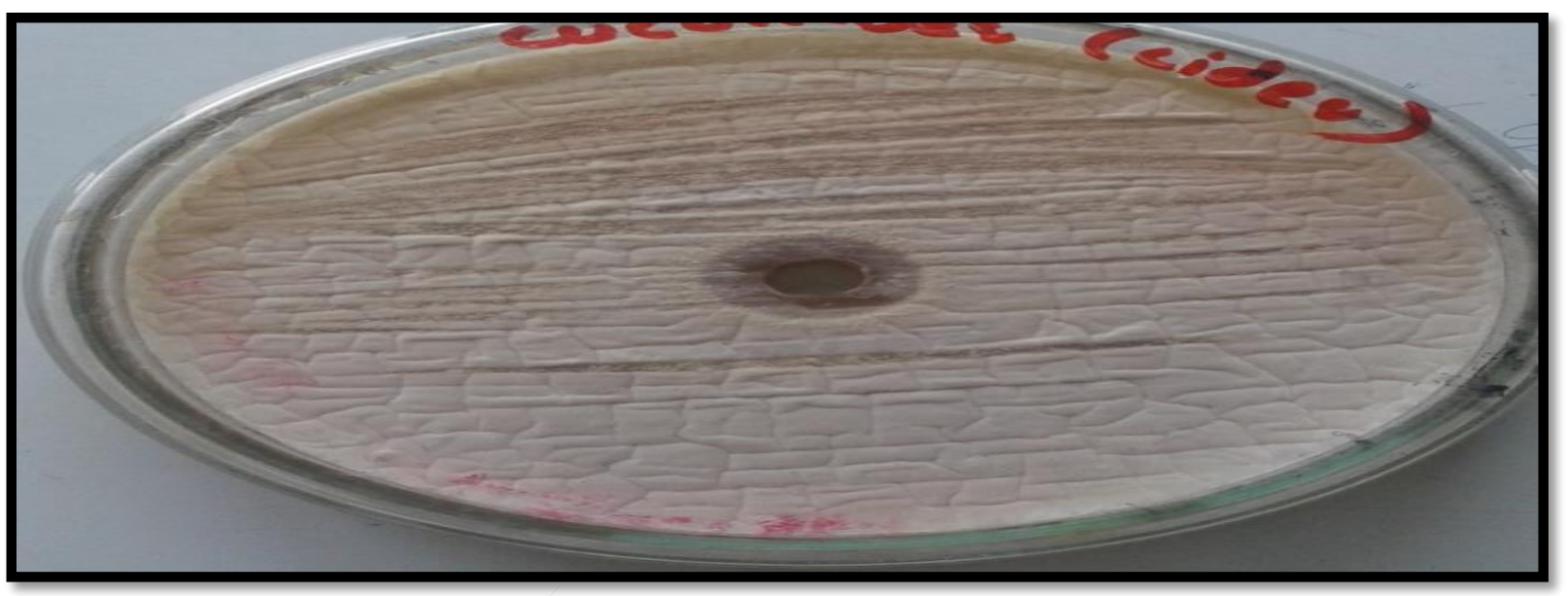

Figure 7. Grape vinegar showed activity against $S$. kiliense by forming zone of inhibition of $0.48 \pm 0.05 \mathrm{~mm}$ after 72 hours

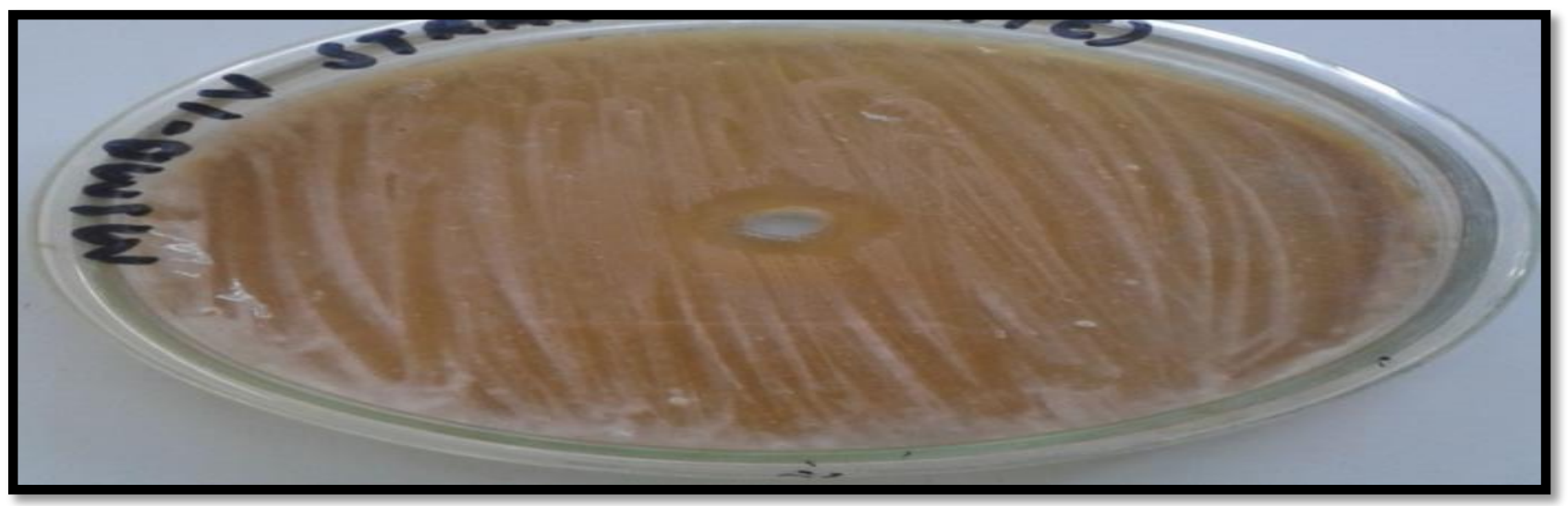

Figure 8. Apple cider vinegar showed activity against $S$. kiliense by forming zone of inhibition of $0.4 \pm 0.05 \mathrm{~mm}$ after 72 hours 


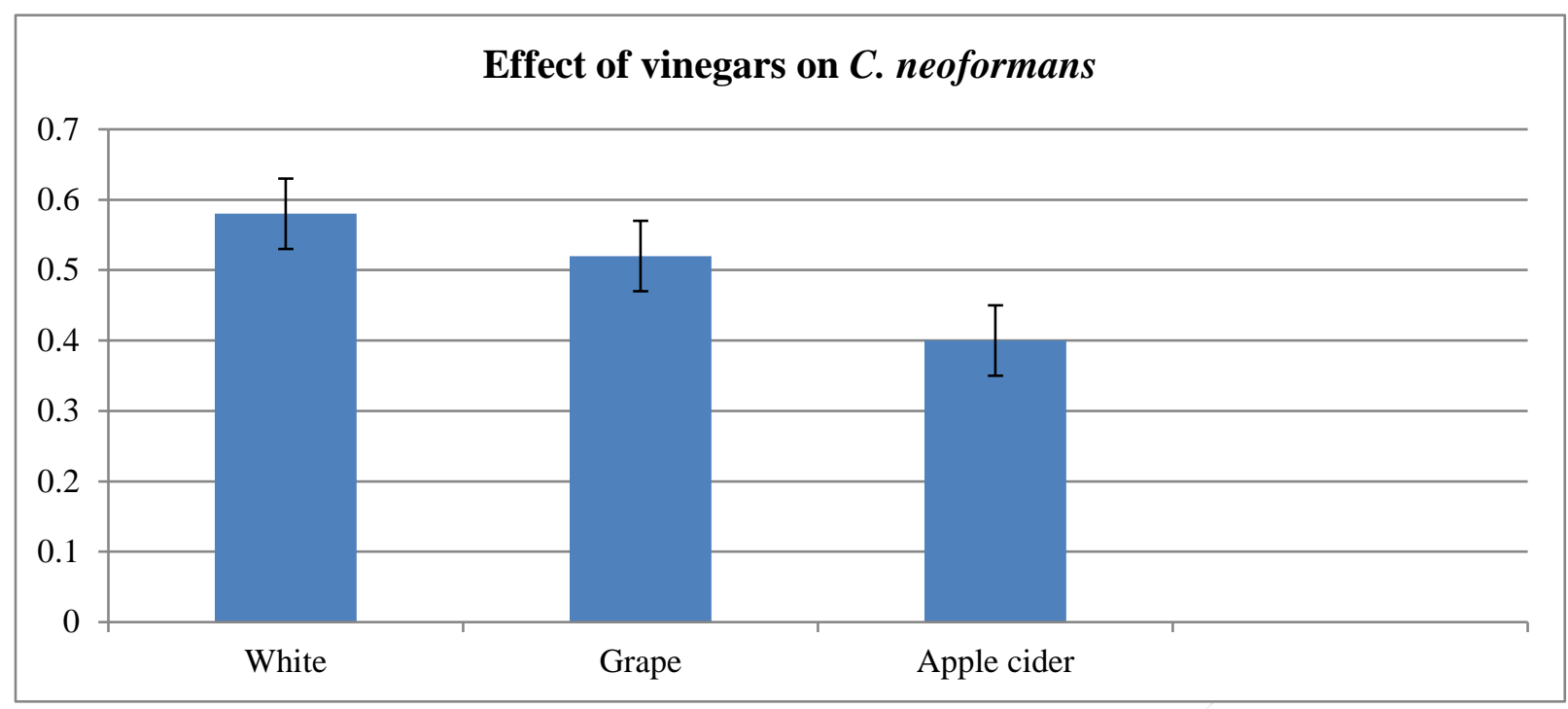

Figure 9. Shows the best effect of distilled white vinegar against $C$. neoformans with mean value of $0.58 \pm 0.05 \mathrm{~mm}$. The moderate effect was observed for grape and least for apple cider with the mean value of $0.4 \pm 0.05 \mathrm{~mm}$

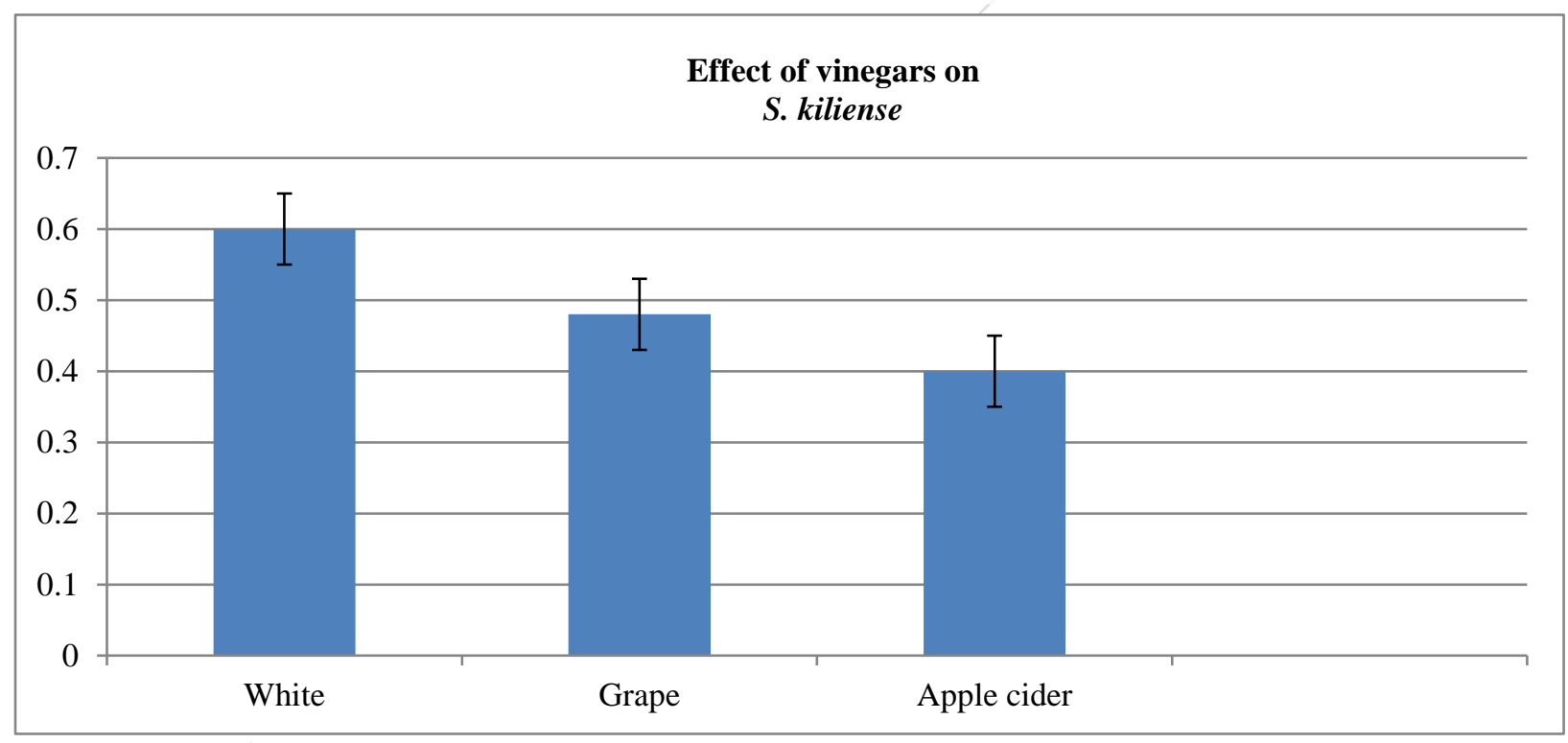

Figure 10. showing the zones of inhibition of vinegars against $S$. kiliense. Distilled white has the best effect against fungal species showing zone of inhibition of $0.6 \pm 0.05 \mathrm{~mm}$

Table 1. Showing the varying zone of inhibition against fungal species

\begin{tabular}{|c|c|c|c|}
\hline \multicolumn{4}{|c|}{ Vinegars showing zone of inhibition (mm) } \\
\hline Fungal species & Distilled white & Grape & Apple cider \\
\hline C. neoformans & $0.58 \pm 0.05$ & $0.52 \pm 0.05$ & $0.4 \pm 0.05$ \\
\hline S. kiliense & $0.6 \pm 0.05$ & $0.48 \pm 0.05$ & $0.4 \pm 0.05$ \\
\hline
\end{tabular}




\section{Discussion}

Vinegars have been used as antifungals from ancient times to preserve food from fungal and bacterial pathogens. The key component in vinegar is acetic acid which has antifungal activity. The use of vinegars to cure infections is reported since the time of Hippocrates. A combination of honey and vinegar was also used to treat cough in ancient times. This study is also conducted to evaluate antifungal activity of different types of vinegars. Vinegars are used against fungus in various applications which has been demonstrated to prevent the germination of conidia of vegetable decaying fungi. Vinegar has showed antifungal properties against conidia of fungi growing on fruits. Hence it is a known antimicrobial agent and there is evidence to suggest that is has some antifungal properties [12].

In the present study, $C$. neoformans and $S$. kiliense have been isolated from vegetable source i.e. rotten cucumber and tomato. Three types of vinegars named distilled white vinegar, grape vinegar and apple cider have been utilized against the growth of fungus to test their antifungal potential. Vinegars showed significant zone of inhibition of different diameters against fungal specie indicating their effectiveness.

Our results showed prominent zones of inhibition against the fungal specie meaning these vinegars have best antifungal activities. Among all vinegars, white distilled vinegar has better antifungal activity against both fungal isolates i.e. $C$. neoformans and $S$. kiliense. While the grape vinegar and apple cider also showed activities against fungal isolates.

No literature has been found in which apple cider, grape vinegar and white synthetic vinegar have applied against fungal species e.g. C. neoformans and S. kiliense.

\section{Conclusion}

Although, traditionally vinegars have been used as food flavoring and preservative agents, but recent studies have shown that the active components present in vinegars are beneficial for human health as well e.g. maleic acid, phenolics and flavonoids are antifungals as well as antioxidants. In the present study, vinegars are used against fungi namely $C$. neoformans and $S$. kiliense. The resultant antifungal activity of vinegars showed that they can be used as an alternative to synthetic antifungal agents. Phenolic compounds in vinegar can scavenge superoxide anion and free radicals in vivo resulting in a potent antioxidant activity.

\section{Authors' contributions}

Conceived and designed the experiments: A Imtiaz \& MA Khan, Performed the experiments: $\mathrm{H}$ Niamat \& Muneeba, Analyzed the data: A Imtiaz \& H Niamat, Contributed materials/ analysis/ tools: Department of Biology, Lahore Garrison University, Lahore, Wrote the paper: $\mathrm{H}$ Niamat \& A Imtiaz.

\section{References}

1. Nascimento M, Silva N, Catanozi M \& Silva K (2003). Effects of different disinfection treatments on the natural microbiota of lettuce. $J$ of Food Prot 66(9): 1697-1700.

2. Kwon-Chung KJ, Edman JC \& Wickes BL (1992). Genetic association of mating types and virulence in Cryptococcus neoformans. Infec and Imm 60(2): 602-605.

3. Levitz SM \& Tabuni A (1991). Binding of Cryptococcus neoformans by human cultured macrophages. Requirements for multiple complement receptors and actin. J of Clin Invest 87(2): 528.

4. Lis-Balchin M \& Deans S (1997). Bioactivity of selected plant essential oils against Listeria monocytogenes. $J$ of Appl Microbiol 82(6): 759-762.

5. Rantsiou K, Greppi A, Garosi M, Acquadro A, Mataragas M \& Cocolin L (2012). Strain dependent expression of stress response and virulence genes of 
Listeria monocytogenes in meat juices as determined by microarray. Inter $J$ of Food Microbiol 152(3): 116-122.

6. Morales-López SE, Parra-Giraldo CM, Ceballos-Garzón A, Martínez HP, Rodríguez GJ, Álvarez-Moreno CA \& Rodríguez JY (2017). Invasive infections with multidrug-resistant yeast Candida auris, Colombia. Emer Infect Dis 23(1): 162.

7. Scott PA, Hansell RI \& Fayle DC (1987). Establishment of white spruce populations and responses to climatic change at the treeline, Churchill, Manitoba, Canada. Arctic and Alpine Res 19(1): 45-51.

8. Franzot SP, Salkin IF \& Casadevall A (1999). Cryptococcus neoformans var. grubii: separate varietal status for Cryptococcus neoformans serotype A isolates. J of Clin Microbiol 37(3): 838840.

9. Burt SA \& Reinders RD (2003). Antibacterial activity of selected plant essential oils against Escherichia coli O157: H7. Letters in Appl Microbiol 36(3): 162-167.

10. Ray CA, Black RA, Kronheim SR, Greenstreet TA, Sleath PR, Salvesen GS \& Pickup DJ (1992). Viral inhibition of inflammation: cowpox virus encodes an inhibitor of the interleukin- $1 \beta$ converting enzyme. Cell 69(4): 597-604.

11. Stehmann JR, Forzza RC, Salino A, Sobral M, Costa Dd \& Kamino LHY (2009). Plantas da Floresta Atlântica 1.

12. Sholberg $\mathrm{P}$, Haag $\mathrm{P}$, Hocking $\mathrm{R} \&$ Bedford K (2000). The use of vinegar vapor to reduce postharvest decay of harvested fruit. Hort Sci 35(5): 898-903. 